\title{
Applications of EBSD to the Study of Localized Deformation*
}

\author{
R.H. Geiss, D.T. Read, A. Roshko**, K.A. Bertness**, and R.R. Keller \\ NIST, Materials Reliability Division, 325 Broadway, Boulder, CO 80305 \\ **NIST, Optoelectronics Division, 325 Broadway, Boulder, CO 80305
}

The use of electron backscatter diffraction (EBSD) to study localized deformation in materials under three very different types of strain is presented.

In the first study, the elastic strain associated with selectively oxidized, multilayered AlGaAs/GaAs structures will be discussed wherein the ESBD patterns were analyzed in three different ways. In one, the automatically collected pattern sharpness maps were used to qualitatively reveal the resulting strain field about the oxide growth front, see Figure 1. In another, manually collected diffraction patterns were mathematically manipulated to qualitatively display the strain field. Thirdly, the band widths of individual patterns from unstrained and strained areas were compared to permit a semi-quantitative appraisal of the magnitude of the strain. Finally, for completeness, these results will be compared with strain fields as determined by finite element simulations.

The second example presents a study of deformation in narrow Al-1\%Si interconnects after low frequency, AC cycling at high current density $\left(>10^{12} \mathrm{MA} / \mathrm{m}^{2}\right)$. Joule heating and differential thermal expansion between the aluminum lines and the $\mathrm{SiO}_{2}$ substrate caused cyclic thermal straining, resulting in thermo-mechanical fatigue. By quasi in-situ testing, we determined the evolution of the crystallography of hundreds of grains and boundaries in the interconnect lines. Typical SEM images and corresponding IPF maps for a $20 \mu \mathrm{m}$ segment of a tested line are shown in Figure 2. These results allowed us to formulate a mechanistic understanding of the deformation process, including slip line formation and grain growth. Significant grain rotations, some more than $30^{\circ}$, were observed, especially in grains that showed the greatest deformation. These grains usually deformed to a structure that we refer to as popcorn. There is an apparent correlation between the starting grain orientation and size and its tendency to form popcorn. Traces of (111) slip planes at the film surface are usually not aligned with the observed surface deformation suggesting that slip occurred on multiple slip systems. This is also confirmed by the observation of large angle grain rotations.

In the third example we present EBSD data concerning the progress of individual grains in a quasi in-situ tensile deformation study of an approximately $1 \mu \mathrm{m}$ thick free standing aluminum film. Through careful analysis we determined that there was very little grain rotation throughout the majority of the test section during the deformation. But in the area which ultimately failed in a chisel point fracture, a very high density of slip lines was observed and significant grain rotation measured. In a high magnification EBSD map of the fractured tip, we observed local rotation of about $25^{\circ}$. It follows the classic single slip tensile axis deformation path from an orientation near a $<101>$ toward the $<112>$. The IPF map and pattern for this deformation are shown in Figure 3 . The grains where the fracture occurred were very large and spanned the width of the test section. They were initially oriented with the ND near $<111>$ and the TD near $<101>$.

We acknowledge support from the NIST Advanced Technology Program and the NIST Office of Microelectronics Programs.

* Contribution of U.S. Department of Commerce; not subject to copyright in the U.S.A. 


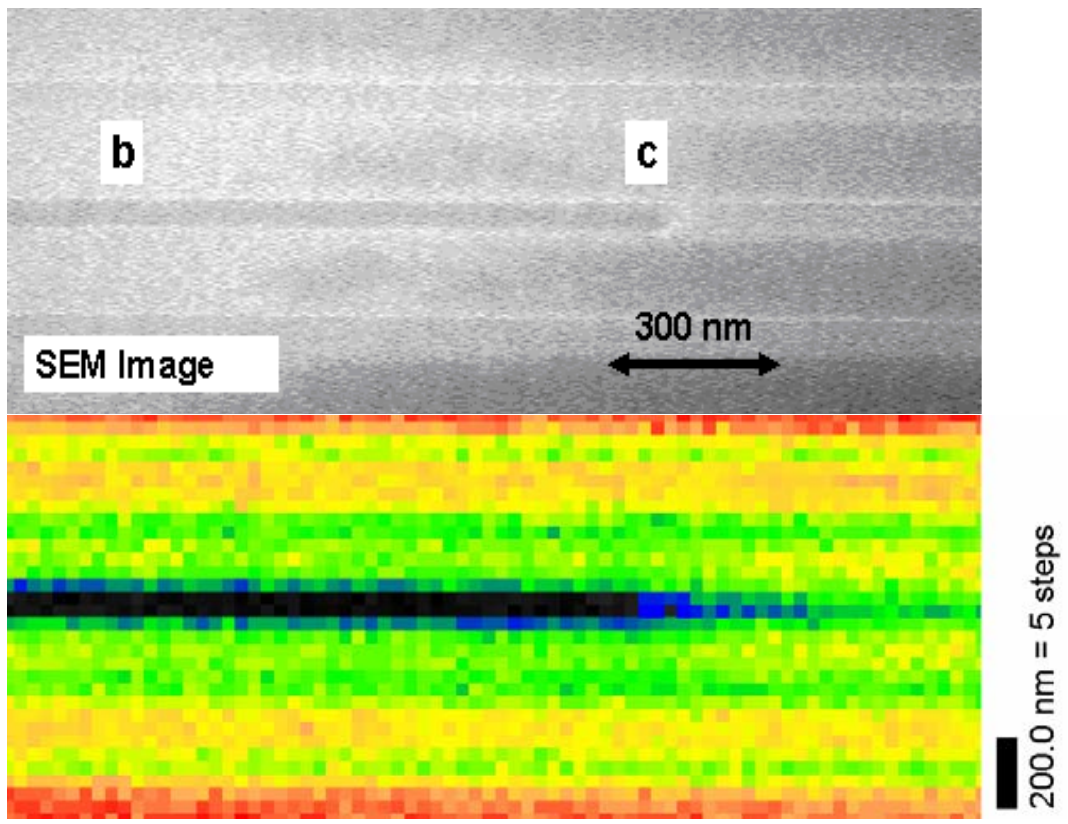

Figure 1.

SEM and pattern sharpness images of the same oxidized growth front.

SEM image is on the left

The pattern sharpness image maps out the strain field using a rainbow in color spectrum, red being the sharpest to blue being the poorest, around the oxidized AlAs growth front, colored black, from $\mathrm{b}$ to $\mathrm{c}$.
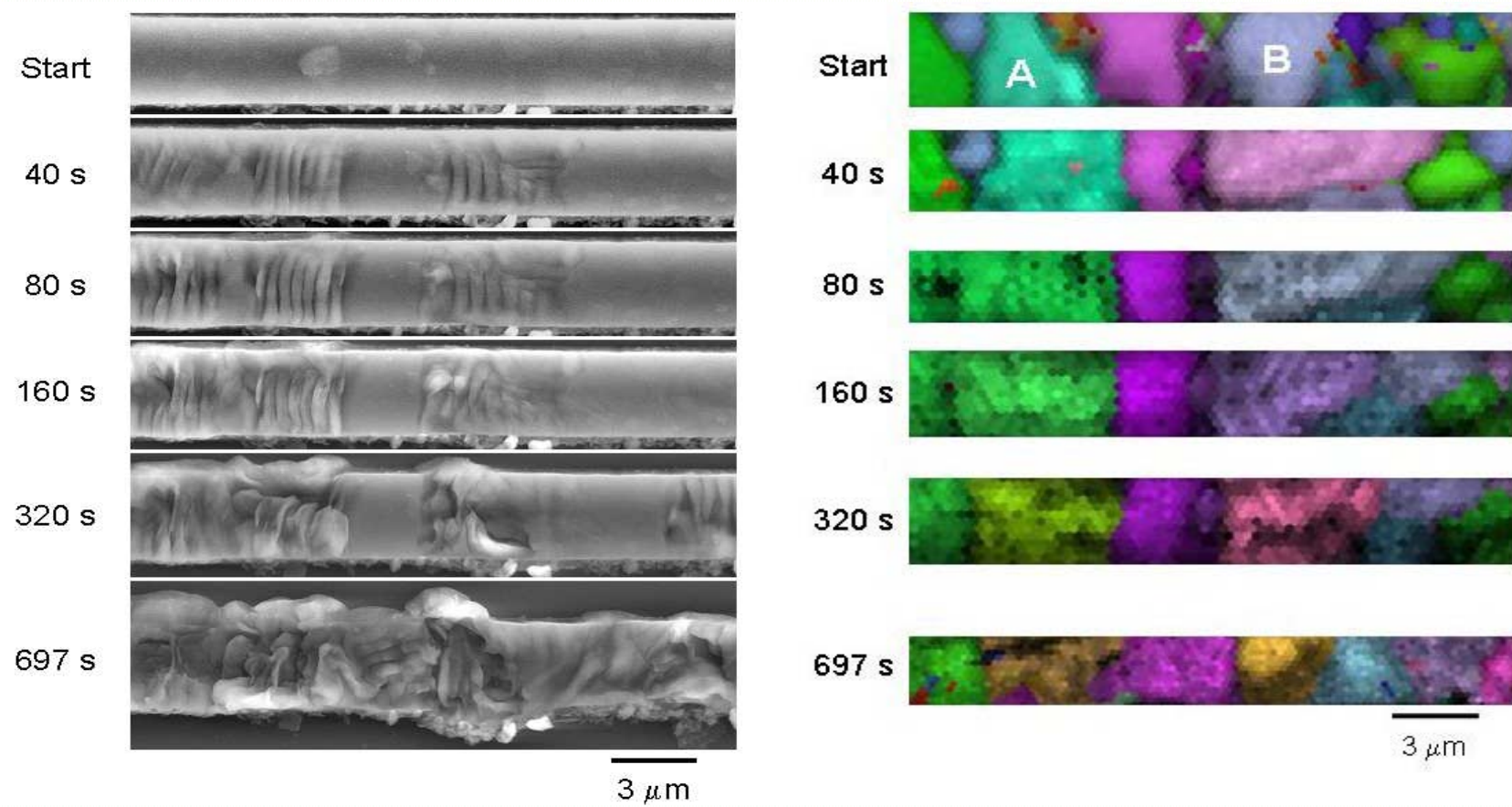

$320 \mathrm{~s}$

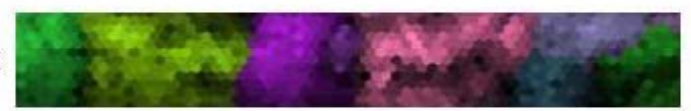

$697 \mathrm{~s}$

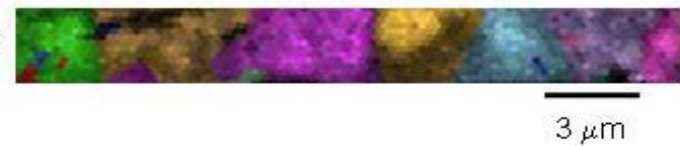

Figure 2. Damage evolution due to thermomechanical AC stressing of an Al-1\% Si line. SEM images on the left show the time progression of the quasi in-situ experiment.

TD-IPF images on the right are orientation maps which correspond to the SEM images.
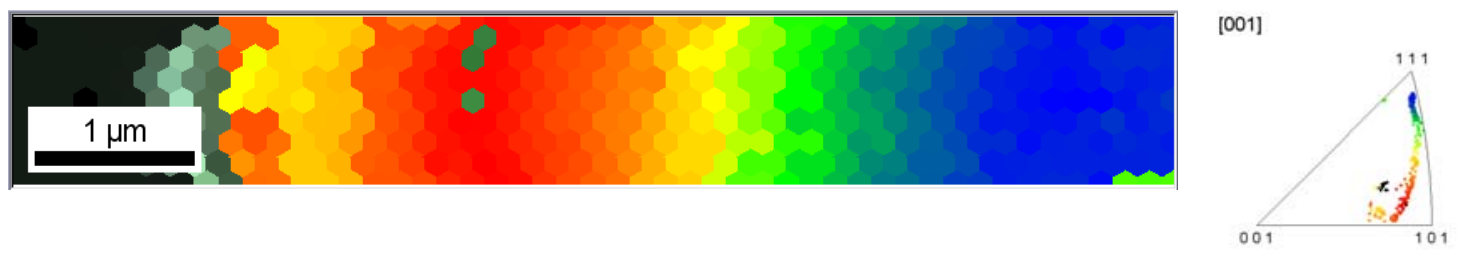

[010]

Figure 3. IPF map colored with a rainbow representation of the orientation of the tensile axis. 